\title{
Incidence of Femoral Stem Subsidence Following Aseptic Hip Revision Using Tapered, Fluted Titanium Stems
}

Thomas J. Baldwin BS, ${ }^{1}$ Evan R. Deckard BSE, ${ }^{2}$ Mary Ziemba-Davis BA, ${ }^{3}$ Leonard T. Buller MD, ${ }^{2,3}$ R. Michael Meneghini MD ${ }^{2,3}$

\author{
${ }^{1}$ Indiana University School of Medicine \\ ${ }^{2}$ Indiana University School of Medicine, Department of Orthopedic Surgery \\ ${ }^{3}$ Indiana University Health Physicians, Orthopedics
}

Background and Hypothesis: Implant design in revision total hip arthroplasty (rTHA) has evolved such that tapered, fluted titanium (TFT) femoral stems have become the gold standard. However, there is a paucity of data on TFT stem subsidence rates following aseptic rTHA. Subsidence can lead to stem loosening, hip instability, leg length discrepancy, and gait impairment in which patients experience great physical challenges requiring undesirable repeat revision surgery. This study assessed the incidence of subsidence in aseptic rTHA performed with TFT stems.

Design/Methods: 102 TFT femoral stems implanted between 2011 and 2020 at a single center were retrospectively reviewed. Stem subsidence was measured on digital radiographic images taken immediately after surgery, at 4-weeks and 1-year postoperatively, and at subsequent follow-up clinic visits. Patient characteristics and risk factors for subsidence, revision etiology, and surgical details (stem type, size, etc.) also were extracted from the electronic medical record.

Results: 102 aseptic rTHAs were analyzed. 21.8\% (22/101) of stems subsided between the immediate postoperative and 4 week follow-up period. $43.1 \%$ $(22 / 51)$ of stems subsided between 4 weeks and 1 year postoperatively, and $39.3 \%(11 / 28)$ of stems subsided between 1 year and latest follow-up (>12 months post-op). At all three time periods, the amount of subsidence was $<3 \mathrm{~mm}$ in a majority of cases ( $77 \%$ to $96 \%$ ) which is considered to be an acceptable amount of stem subsidence or "settling." Only seven TFT stems had subsided more than $3 \mathrm{~mm}$. Two of these stems subsided 10 or more $\mathrm{mm}$ but had not required revision at latest follow-up of 4 and 8 years.

Conclusion and Potential Impact: The incidence of significant TFT stem subsidence requiring repeat revision surgery was relatively low in this cohort. Further research is warranted to identify specific risk factors for subsidence of TFT femoral stems. 